\title{
Expanding the ICA model of stakeholders in a spatial data in- frastructure (SDI)
}

\author{
Antony K Cooper ${ }^{a, b *}$, Serena Coetzee ${ }^{b}$, Petr Rapant ${ }^{\mathrm{c}}$, Adam Iwaniak ${ }^{\mathrm{d}}$, Jan Hjelmager ${ }^{\mathrm{e}}$, Harold \\ Moellering ${ }^{\mathrm{f}}$, Michel Huet ${ }^{\mathrm{g}}$ and Kisco Sinvula ${ }^{\mathrm{h}}$
}

${ }^{a}$ Smart Places, CSIR, Pretoria, South Africa, acooper@csir.co.za

${ }^{b}$ Centre for Geoinformation Science, Department of Geography, Geoinformatics and Meteorology, University of Pretoria, Pretoria, South Africa, serena.coetzee@up.ac.za

${ }^{c}$ Institute of Geoinfomatics, VSB-Technical University of Ostrava, Ostrava-Poruba, Czech Republic, petr.rapant@vsb.cz

${ }^{d}$ Wroclaw Institute of Spatial Information and Artificial Intelligence, Wroclaw, Poland, adam.iwaniak@wizipisi.pl

${ }^{e}$ Danish Agency for Data Supply and Efficiency, Copenhagen, Denmark, jnh@sdfe.dk

${ }^{f}$ Department of Geography, Ohio State University, Columbus, OH, 43210,USA, geohal+@osu.edu

${ }^{g}$ Retired, michel.m.huet@neuf.fr

${ }^{h}$ Kunene Regional Council, Windhoek, Namibia, ksinvula@gmail.com

* Corresponding Author

Keywords: spatial data infrastructure, SDI, stakeholder, ICA model

\begin{abstract}
\section{Spatial data infrastructure}

No mapping agency can expect to capture and process entirely by itself, all the geospatial data needed for its products. The agency needs data sets from elsewhere and workflows and protocols for creating its various products. Unsurprisingly, such workflows and inter-institutional arrangements have evolved into broader collaborations, particularly as spatial data infrastructures (SDIs). An SDI is an evolving concept about facilitating and coordinating the exchange and sharing of geospatial data and services between stakeholders from different levels in the geospatial data community.
\end{abstract}

The Commission on SDI \& Standards (and its predecessors) of the International Cartographic Association (ICA) has developed formal models of an SDI, using the viewpoints of the Reference Model for Open Distributed Processing (RM-ODP) and the Unified Modeling Language $(U M L)$ for the detailed modelling. The Commission described an SDI from the Enterprise Viewpoint (purpose, scope and policies for an SDI), Information Viewpoint (semantics of information and information processing in an SDI) and Computational Viewpoint (functional decomposition of the SDI into a set of services that interact through interfaces) (Hjelmager et al., 2008, Cooper et al., 2012). The ICA Commission did not investigate the Engineering and Technology Viewpoints of an SDI, because they are implementation-specific and the Commission has aimed at providing technology-independent models for an SDI. Other researchers have used the Engineering and Technology Viewpoints for specific SDIs.

\section{SDI stakeholders}

The Enterprise Viewpoint model (Hjelmager et al., 2008) included six types of stakeholders in an SDI: Policy Maker, Producer, Provider, Broker, Value-added Reseller and End User. Any one person, group, committee or organisation can have multiple stakeholder roles, which could be simultaneously. Each stakeholder can have an active or passive relationship with any activities or components in an SDI. A stakeholder in an SDI could also be considered to be an actor (Oliveira and Lisboa-Filho, 2015). Various trends are increasing the number of stakeholders in the SDI, their associated diversity and heterogeneity, and the resources at their disposal.

Subsequently, the ICA Commission assessed whether or not these SDI models catered for volunteered geographical information (VGI), that is, user-generated geospatial content, or user-generated content with geospatial components. The models are sufficiently robust to do so, though it was then realised that the SDI stakeholders needed to be described in more detail as subtypes of the general roles of the six types of SDI stakeholders. The Commission then identified 24 subtypes for the stakeholders, with several of these subtypes having further sub-subtypes. For example, a Producer could have a subtype Status, which in turn could have the subtypes Official Mapping Agency or Commercial Mapping Agency (Cooper et al., 2011). However, a better term than subtype might be specialization, special case, attribute, activity or role.

Collectively, these Enterprise, Information and Computational Viewpoints and the detailed stakeholders form what is known colloquially in the literature as the ICA model or ICA's formal model of an SDI, such as by (Oliveira et al., 2016). The ICA SDI model is not meant to be a prescriptive model of an SDI, but rather a descriptive one. The ICA SDI model is also implementation-independent and hence somewhat abstract.

\section{Issues with the stakeholders}

While all the stakeholder subtypes in an SDI were given definitions, some of the types and subtypes were confused with one another by other researchers. Further, it became clear that not all stakeholders have benevolent relationships with SDIs, whether the stakeholders be malevolent, 
or just too idle or incompetent to help the SDI succeed. Hence, it is necessary to update the ICA SDI stakeholder model.

We have reviewed the literature and several authors have proposed improvements to the ICA SDI stakeholder model and have highlighted parts of the model that are not well understood. Further, the ICA Commission itself has also revisited the model at previous meetings. The key contributions concerning the stakeholder model are summarised in this paper. They include:

- Making it clear that any contributor to an SDI should control their assets in the SDI and can withdraw them when they see fit.

- Catering explicitly for a contact or representative for any community.

- Including an educator role, and by extension, a researcher role.

- Including a role for those who fund the SDI itself and fund all of the relevant stakeholders so that they can function effectively.

- Catering for how stakeholders could be assembled together, such as in special-interest groups.

- Catering for oversight explicitly, such as through an ombud.

- Making it clear that a subtype of a stakeholder is not necessarily subordinate to that stakeholder.

- Characterising the maturity of a stakeholder.

- Including a subtype for public-sector producers that are not official mapping agencies.

- Whether or not agency, contracting or representation roles or functions should be deemed to be separate stakeholders in the model.

- Improving the End User, which has only two subtypes.

- Catering for shifts in the roles and characteristics of SDI stakeholders over time.

- Including service producers explicitly.

- Catering for representatives of the SDI who liaise with other organisations or other SDIs.

- Including explicitly some of the roles within the Secretariat, such as systems administration, technical support and quality assurance.

- Some attributes could be common across all or most of the six types of stakeholders in an SDI, such as their motivation for contributing to, or using, any particular SDI; their authority or ability to contribute; whether or not they are liable for their contributions and any consequences thereof; and the ethics related to the contribution, including invasion of privacy, arbitrary restrictions or mischief.

- The SDI stakeholder model needs to cater for both the top-down and the bottom-up approaches to an SDI.

This paper proposes some revisions and expansions on this ICA model of the stakeholders in a spatial data infrastructure. While updating the model has not yet been completed, we believe that it will be appropriate to present our findings now to the ICA community to obtain feedback.

\section{Acknowledgements}

Some of the work described herein was facilitated by funding from the International Cartographic Association (ICA) through the Commission on SDI \& Standards, such as for the workshop in Dresden. We would also like to thank our employers for allowing us to participate in this research.

\section{References}

Cooper, A. K., Moellering, H., Hjelmager, J., Rapant, P., Delgado, T., Laurent, D., Coetzee, S., Danko, D. M., Düren, U., Iwaniak, A., Brodeur, J., Abad, P., Huet, M. and Rajabifard, A., 2012. A spatial data infrastructure model from the computational viewpoint. International Journal of Geographical Information Science 27(6), pp. 11331151 .

Cooper, A. K., Rapant, P., Hjelmager, J., Laurent, D., Iwaniak, A., Coetzee, S., Moellering, H. and Düren, U., 2011. Extending the formal model of a spatial data infrastructure to include volunteered geographical information. In: 25th International Cartographic Conference (ICC 2011), Paris, France.

Hjelmager, J., Moellering, H., Delgado, T., Cooper, A. K., Rajabifard, A., Rapant, P., Danko, D., Huet, M., Laurent, D., Aalders, H. J. G. L., Iwaniak, A., Abad, P., Düren, U. and Martynenko, A., 2008. An initial formal model for spatial data infrastructures. International Journal of Geographical Information Science 22(11), pp. 1295-1309. Oliveira, I. L. and Lisboa-Filho, J., 2015. A spatial data infrastructure review - sorting the actors and policies from enterprise viewpoint. In: 17th International Conference on Enterprise Information Systems (ICEIS 2015), Barcelona, Spain, pp. 287-294.

Oliveira, I. L., Lisboa-Filho, J., Moura, C. A. and da Silva, A. G., 2016. Especifying the enterprise and information viewpoints for a corporate spatial data infrastructure using ICA's formal model. In: 18th International Conference on Enterprise Information Systems (ICEIS 2016), Rome, Italy, pp. 271-282. 